\title{
THE PATIENT WITH DEPRESSION: ASSESSMENT AND MANAGEMENT
}

Dr Tan Zhenwen Tina

\begin{abstract}
Depression in adults is a condition that is treatable by family physicians. The current Clinical Practice Guidelines from the Ministry of Health recommends a selective serotonin reuptake inhibitor (SSRI) as the first-line of pharmacotherapy. Care should be taken to assess the patient's psychological and social factors contributing to the illness. These factors should be managed by referring the patient to an appropriate allied health professional, such as a psychologist or communitybased social worker. Specialist referral should be made under certain circumstances (such as treatment-resistance), or if issues pertaining to risk arise. This review aims to give an update on the recently published DSM-5 criteria for diagnosis, and the treatment of the adult patient with depression.
\end{abstract}

Keywords: Depression; DSM-5; SSRIs; antidepressants; non-pharmacological treatment

\section{SFP2019; 45(5) : 13-16}

\section{INTRODUCTION}

Depression is largely considered the "common cold" of mental health problems. The World Health Organization (WHO) has ranked this condition as the single largest contributor to global disability. ${ }^{1}$ It is estimated that 4.4 percent of the global population suffers from depression, which translates to an estimated 300 million people worldwide, making this condition the leading cause of disability worldwide. ${ }^{2}$

While a certain proportion of patients can recover from depression without treatment, the majority would have a chronic and intermittent course. A recent primary care study showed that 43 percent of patients recovered without treatment, but those with a chronic course of illness had higher levels of depressive symptoms, somatic symptoms and greater mental dysfunction at baseline. ${ }^{3}$

Chronic depression also increases a person's suicide risk, and is associated with serious impairment in quality of life, and has major economic impact owing to occupational costs, medical service costs, and suicide-related costs. ${ }^{4}$

The Singapore Mental Health Study, which was conducted in 2010, showed that the lifetime prevalence of Major Depressive Disorder was 5.8 percent $^{5}$, with a higher prevalence among women than men.

\section{Dr Tan Zhenwen Tina}

Consultant, Institute of Mental Health
There is therefore a role for family physicians to assess and treat patients with depression. The patient may be more likely to seek help from their family physician for various reasons. These include the perceived reduced stigma of being treated by a primary care doctor, as well as the familiarity that a patient may have with their family physician. Combined with the responsiveness of depression to medications and psychological therapy, this place family physicians in an ideal position to diagnose and treat patients with depression.

The focus of this review will be on the assessment of depression in adult patients, and the treatment options (pharmacological and non-pharmacological) available to family physicians. The duration of pharmacological treatment needed, and indications for specialist referral, will also be discussed.

\section{DSM-5 DIAGNOSTIC CRITERIA}

In 2013, the American Psychiatric Association published the Fifth Edition of the Diagnostic and Statistical Manual of Mental Disorders (DSM-5). The diagnostic criteria of Major Depressive Episode(MDE) is largely unchanged from previous versions of the DSM (Table 1), except for changes in episode specifiers, which aimed to better reflect the spectrum of clinical presentations in Major Depressive Disorder (MDD)such as MDD “with anxious distress".

The diagnostic criteria also continue to allow for the severity of the current episode to be stated (Table 1), which would enable the family physician to determine the level of treatment that a patient requires.

\section{ASSESSMENT}

Depression can present in a variety of ways, especially in the primary care setting. Patients may attribute emotional difficulties to work or other life stressors, or may present with predominantly physical symptoms. It is useful to be prepared to assess such patients more thoroughly, if indicated.

Screening for depression can be done in primary care settings in individuals with risk factors, provided resources are available for subsequent management and follow-up.,6 Risk factors for depression would include those with a personal history of depression, family history of depression, and/or chronic medical problems. The Canadian Network for Mood and Anxiety Treatments (CANMET) has recommended a 2-question screen ${ }^{4,7}$ as follows:

1. In the last month, have you been bothered by little interest or pleasure in doing things?

2. In the last month, have you been feeling down, depressed or hopeless? 
An answer of "yes" to either question should be followed by a more detailed assessment. This includes a biopsychosocial assessment, mental state examination, and physical examination. ${ }^{6}$

The basic assessment for depression begins with a detailed history of the presenting symptoms, the severity, level of functional impairment (including life events and stressors), and duration of the current episode. A brief screen for prior depressive episodes, manic or hypomanic episodes, substance use and pre-existing medical conditions should be included. The patient's personal history, including social background,past psychiatric illness, and family history of mental illness, depression and suicide should be established.

In this regard, time should be spent on establishing therapeutic alliance with the patient, determining life stressors as well as sources or support and personal resources. Collateral information should be gathered where possible.

A mental state examination should be included as part of the assessment. This includes an evaluation of the severity of symptoms and a screen for psychotic symptoms. It is also necessary to establish the risk of suicide, self-harm, and risk of harm to others.

A physical examination (and indicated laboratory testing) is indicated to rule out a medical or surgical condition that may cause similar symptoms.

\section{Assessing suicide risk}

Major depression is a risk factor for suicide, accounting for up to 60 percent of suicides. Also, such persons are more likely to visit their primary care practitioners than a psychiatrist. One of the tools used to assess suicide risk is the SAD PERSONS Scale. ${ }^{14}$ This is a ten-item mnemonic with a maximum score of ten points. Close monitoring of patients is needed for scores of three to four; strongly consider hospitalisation for those with scores of five and six; and hospitalisation for further assessment for patients with scores of seven to ten.

\section{PHARMACOLOGICAL TREATMENT}

Depression is a highly treatable condition, and meta-analyses continue to show the efficacy of antidepressants in treating adults with this condition. ${ }^{8,9}$

In the acute phase of treatment, which lasts from eight to twelve weeks, the primary goal is to eliminate all signs and symptoms of depression, and restore the patient to their premorbid level of functioning. ${ }^{4,6}$ It is important to aim for full symptom remission, because residual depressive symptoms increase the risk of relapse and worsens the long-term prognosis. Medications should be titrated until appropriate therapeutic doses, or if further referral is needed (in cases where symptoms are not responding to treatment, or if augmentation strategies are indicated).

The selective serotonin reuptake inhibitors (SSRIs), and newer antidepressants remain the first-line drug of choice for pharmacotherapy in depression due to better tolerance, safety in overdose, and favourable risk-benefit ratio., ${ }^{6,10,11}$ Newer antidepressants include serotonin and nor epinephrine reuptake inhibitors (SNRIs), and noradrenergic and specific serotonergic antidepressants (NaSSA).

Ultimately, the choice of antidepressant should be tailored to the patient's profile, symptoms, and personal preference. Consideration should be made into the patient's comorbid medical conditions, potential drug-drug interactions, pertinent side effects and patient's tolerability towards these effects, cost of medication, and previous use of antidepressant, if any. ${ }^{6,11}$ Older antidepressants such as tricyclic antidepressants (TCAs) and monoamine oxidase inhibitors (MAOIs) should not be used as first-line pharmacological treatment due to potential side effects and dangers in overdose. ${ }^{10}$

Patients should be reviewed frequently following commencement of antidepressants, to monitor compliance, side effects and improvement in symptoms. Dosage of medication should be titrated according to symptoms and tolerability. ${ }^{10}$ The frequency of monitoring would depend on the severity of the depressive episode, suicide risk, patient's willingness to come for follow-up, and availability of social support. ${ }^{6}$

Even with treatment, suicidal thoughts, plans and attempts are prevalent among patients with depression. Therefore, every clinical encounter should include an assessment of suicide risk. This is especially important in view of "black box warnings" issued by many regulatory agencies, on the increased suicidality associated with antidepressants, particularly in adolescents and young adults. ${ }^{11}$

Once the patient has been stabilized on a particular dose of antidepressant, and depressive symptoms begin to respond and remit, maintenance treatment should be considered. In this phase, the primary goal would be to prevent recurrence. ${ }^{6}$ This phase would require monitoring and continuation of medications from 6 to 24 months, or longer, depending on the patient's risk factors for recurrence. ${ }^{4}$ Such risk factors would include past episodes of depression (indicating a risk of recurrence), an earlier age of onset, poor social support, and ongoing psychosocial stressors.

Patients should be advised on the risk of symptom recurrence, or a full relapse, if medications were inappropriately down-titrated or discontinued. There is also a risk of discontinuation reaction, if treatment were abruptly stopped. ${ }^{6,10}$ However, if the patient is stable and well after an appropriate period of maintenance, discontinuation can be discussed, taking into account the patient's current psychosocial situation and readiness to stop medication. A gradual tapering of antidepressant is recommended, at a minimum of four weeks or longer, to reduce the side effects of discontinuation. The patient should also be advised that the highest risk of relapse would be during the initial six months after medication is completely stopped. ${ }^{10}$

\section{NON-PHARMACOLOGICAL APPROACHES}

\section{Psychological treatment}

Psychological treatment remains a viable option, allowing the patient to establish treatment alliance with a trained therapist, alleviate psychological symptoms of depression, and prevent or 
correct maladaptive patterns of behaviour. ${ }^{6}$ It is an established form of treatment ${ }^{12,13}$, and can be used as first-line treatment alone, in cases of mild to moderate depression. ${ }^{6}$ Psychotherapy should not be used in patients with severe symptoms, such as psychomotor retardation; instead, such patients would benefit from pharmacological treatment.

The decision for psychotherapy should be determined by the patient's choice, his/her suitability to undergo psychotherapy (ability to be self-aware of emotions and to express themselves), and the availability of a therapist. ${ }^{6,10}$ Psychotherapy may be of particular benefit in patients with significant psychosocial stressors, interpersonal difficulties, intrapsychic conflict, or have a comorbid personality disorder. ${ }^{6}$

The form and duration of psychotherapy may be determined by the therapist whom the patient seeks for psychological treatment. However, the patient should continue to be reviewed by the treating physician on a regular basis to evaluate improvement of symptoms. If there is no or partial response after four to eight weeks of psychotherapy, antidepressants should be considered. ${ }^{6}$

\section{Psychoeducation and family intervention ${ }^{6}$}

During the course of treatment, it is important to continually educate the patient on the illness, the need for adherence to treatment, and to instil in the patient a hope for recovery. Lifestyle advice such as exercising, stress reduction, and strategies on reducing anxiety, should also be part of the psychoeducation process.

Family members or trusted friends in the patient's circle of social support, can be involved in the patient's treatment and recovery. This is particularly important where there is a risk of suicide.

Where indicated, patients can be referred to the community or Family Service Centres (FSC) for individual counselling to develop coping strategies to help with immediate issues and stressors. Couples/marital therapy may be warranted, especially if marital distress is contributing to the patient's depressive symptoms. A referral to a community-based social worker may also be indicated to assist the patient or family in managing ongoing psychosocial issues, such as financial difficulties.

\section{Physical treatment}

Several forms of physical treatments for depression exist, including electro convulsive therapy (ECT), repetitive transcranial magnetic stimulation (rTMS), and transcranial direct current stimulation (tDCS). However, these are not considered first-line modes of treatment for depression ${ }^{10}$, and further referral to specialist or a facility with the appropriate expertise and equipment is recommended.

\section{FURTHER REFERRAL ${ }^{6}$}

A referral to a psychiatrist and/or an appropriate medical/ psychiatric facility should be considered under these circumstances:

- Diagnostic difficulty

- Treatment resistance (patient does not respond to one to two trials of medication, or needs combination therapy)

- Complex psychosocial / medico-legal issues

- If the depressive episode is associated with high suicide risk
- If symptoms are suggestive of psychosis or mania emerge

- Severe perinatal cases, especially if there is risk of harm to the child

- Co-morbid medical conditions with potential drug-drug interactions

- Comorbid substance abuse

- Any other situation where more urgent or intensive treatment is required, such as an admission or electro convulsive therapy (ECT).

\section{CONCLUSIONS}

Depression is a highly treatable condition that is responsive to antidepressants and psychotherapy. It is important to address psychosocial stressors that have contributed to, and which may perpetuate, a patient's depressive symptoms. The patient should be regularly reviewed to ensure there is response to treatment, and further appropriate referrals to a specialist can be made if indicated.

\section{Table 1: DSM-5 Symptom criteria for Major Depressive Episode}

A. Five (or more) of the following symptoms have been present during the same two-week period and represent and change from previous functioning; at least one of the symptoms is either (1) or (2):

1. Depressed mood most of the day, nearly every day, as indicated by either subjective report (e.g., feels sad, empty, hopeless) or observation made by others (e.g., appears tearful). Note: In children and adolescents, can be irritable mood

2. Markedly diminished interest or pleasure in all, or almost all, activities most of the day, nearly every day (as indicated by subjective account or observation)

3. Significant weight loss when not dieting or weight gain (e.g., a change of more than five percent of body weight in a month) or decrease or increase in appetite nearly every day. Note: In children, consider failure to make expected weight gains

4. Insomnia or hypersomnia nearly every day

5. Psychomotor agitation or retardation nearly every day (observable by others, not merely subjective feelings of restlessness or being slowed down)

6. Fatigue or loss of energy nearly every day

7. Feelings of worthlessness or excessive or inappropriate guilt (which may be delusional) nearly every day (not merely self-reproach or guilt about being sick)

8. Diminished ability to think or concentrate, or indecisiveness, nearly every day (either by subjective account or as observed by others)

9. Recurrent thoughts of death (not just fear of dying), recurrent suicidal ideation without a specific plan, or a suicide attempt or a specific plan for committing suicide

B. The symptoms cause clinically significant distress or impairment in social, occupational, or other important areas of functioning.

C. The episode is not attributable to the physiological effects of a substance or to another medical condition.

D. The occurrence of the major depressive episode is not better explained by schizo affective disorder, schizophrenia, schizophreniform disorder, delusional disorder, or other specified and unspecified schizophrenia spectrum and other psychotic disorders.

E. There has never been a manic episode or hypomanic episode.

- Severity is based on the number of criterion symptoms, the severity of those symptoms, and the degree of functional disability. Mild: Few, if any, symptoms in excess of those required to make the diagnosis are present, the intensity of the symptoms is distressing but manageable, and the symptoms result in minor impairment in social or occupational functioning.

Moderate: The number of symptoms, intensity of symptoms, and/or functional impairment are between those specified for "mild" and severe". Severe: The number of symptoms is substantially in excess of that required to make the diagnosis, the intensity of the symptoms is seriously distressing and unmanageable, and the symptoms markedly interfere with social and occupational functioning. 


\section{REFERENCES}

1. Institute of Mental Health. Coping with depression. [Internet] Singapore: 2012 [Accessed 16 April 2019] Available from: https://www.imh.com.sg/wellness/page. aspx?id=554.

2. UN News. UN health agency reports depression now 'leading cause of disability worldwide?. [Internet]UN:23 Feb 2017.[Accessed 16 April 2019] Available from: https:// news.un.org/en/story/2017/02/552062-un-health-agency-reports-depression-now-leadingcause-disability-worldwide\#.WLRpQBB3xBw.

3. Stegenga BT, Kamphuis MH, King M, Nazareth I, Geerlings MI. The natural course and outcome of major depressive disorder in primary care: the PREDICT-NL study. Social Psychiatry and Psychiatric Epidemiology. 2012 Jan; 47(1):87-95

4. Lam RW, McIntosh D, Wang J, Enns MW, Kolivakis T, Michalak EE, Sareen J, Song WY Kennedy SH, MacQueen GM, Milev RV, Parikh SV, Ravindran AV; CANMAT Depression Work Group. Canadian Network for Mood and Anxiety Treatments (CANMAT) 2016 Clinical Guidelines for the Management of Adults with Major Depressive Disorder: Section 1. Disease Burden and Principles of Care. The Canadian Journal of Psychiatry. 2016 Sep; 61(9):510-23

5. Chong SA, Abdin E, Vaingankar JA, Heng D, Sherbourne C, Yap M, Lim YW, Wong HB, Ghosh-Dastidar B, Kwok KW, Subramaniam M. A population-based survey of mental disorders in Singapore. Annals of Academy Medicine Singapore. 2012 Feb; 41(2):49-66.

6. Chua HC, Chan LL, Chee KS, Chen YH, Chin SA, Chua PL, Fones SL, Fung D, Khoo CL, Kwek SK, Lim EC. Ministry of Health clinical practice guidelines: depression. Singapore Medical Journal. 2012 Feb; 53(2):137-43.

7. Bosanquet K, Bailey D, Gilbody S, Harden M, Manea L, Nutbrown S, McMillan D. Diagnostic accuracy of the Whooley questions for the identification of depression: a diagnostic meta-analysis. BMJ Open. 2015 Dec 9; 5(12): e008913.
8. Cipriani A, Furukawa TA, Salanti G, Chaimani A, Atkinson LZ, Ogawa Y, Leucht S, Ruhe HG, Turner EH, Higgins JPT, Egger M. Comparative efficacy and acceptability of 2 antidepressant drugs for the acute treatment of adults with major depressive disorder: systematic review and network meta-analysis. The Lancet. 2018 Apr 7; 391(10128):1357-66.

9. Hieronymus F, Emilsson JF, Nilsson S, Eriksson E. Consistent superiority of selective serotonin reuptake inhibitors over placebo in reducing depressed mood in patients with major depression. Molecular Psychiatry. 2016 Apr 1;21(4):523-30.

10. Cleare A, Pariante CM, Young AH, Anderson IM, Christmas D, Cowen PJ, Dickens C, Ferrier IN, Geddes J, Gilbody S, Haddad PM. Evidence-based guidelines for treating depressive disorders with antidepressants: a revision of the 2008 British Association for Psychopharmacology guidelines. Journal of Psychopharmacology. 2015 May;29(5):459-525.

11. Kennedy SH, Lam RW, McIntyre RS, Tourjman SV, Bhat V, Blier P, Hasnain M, Jollan F, Levitt AJ, MacQueen GM, McInerney SJ. Canadian Network for Mood and Anxiety Treatments (CANMAT) 2016 clinical guidelines for the management of adults with major depressive disorder: section 3. Pharmacological treatments. The Canadian Journal of Psychiatry. 2016 Sep;61(9):540-60.

12. Cuijpers P, Geraedts AS, van Oppen P, Andersson G, Markowitz JC, van Straten A. Interpersonal psychotherapy for depression: a meta-analysis. American Journal of Psychiatry. 2011 Jun;168(6):581-92.

13. Munder T, Flückiger C, Leichsenring F, Abbass AA, Hilsenroth MJ, Luyten P, Rabung S, Steinert C, Wampold BE. Is psychotherapy effective? A re-analysis of treatments for depression. Epidemiology and psychiatric sciences. 2018 Jul 30:1-7.

14. Ng CW, How CH, Ng YP. Depression in primary care: assessing suicide risk. Singapore medical journal. $2017 \mathrm{Feb}$;58(2):72-7.

\section{LEARNING POINTS}

- SSRIs are the first-line pharmacological treatment for the adult patient with depression. Consideration on which SSRI, or other class of antidepressant to use, should be made based on side effect profile, the patient's preference and depressive symptoms, and comorbid medical conditions.

- A review of psychosocial factors contributing to the patient's illness should be done, and appropriate referrals made to allied health professionals (for example, to a psychotherapist or a marital counsellor).

- Depression is a treatable condition which patients can recover from. Hope should be instilled in them where possible. Specialist referrals can be made if it is indicated. 